\title{
The Study of Optimal Structure and Value of Dump Resistance in Direct-Drive Permanent Magnet Synchronous Generators
}

\author{
Jun Jia, Jie Zhao, Dichen Liu, Chen Cheng, Jun Wang, Linge Qi \\ School of Electrical Engineering, Wuhan University, Wuhan, China \\ Email: jiajuntec@163.com
}

Received February 2014

\begin{abstract}
This paper studied the direct-drive permanent magnet synchronous machine (permanent magnet synchronous generator, PMSG) Chopper optimal topology and resistance value. Compared the different Chopper circuit low voltage ride-through capability in the same grid fault conditions in simulation. This paper computes the dump resistance ceiling according to the power electronic devices and over-current capability. Obtaining the dump resistance low limit according to the temperature resistance allows, and calculating the optimal value by drop voltage in the DC-Bus during the fault. The feasibility of the proposed algorithm is verified by simulation results.
\end{abstract}

\section{Keywords}

\section{Permanent Magnetic Synchronous Generator (PMSG); Optimal Resistance; Chopper}

\section{Introduction}

Direct-drive wind turbines is a kind of generator driven directly by the wind, also known as gearless wind generators, using multi-pole generator motor and impeller driven direct connection, the traditional gear box parts has been eliminated. Because the gearboxes are easily to overload and failure in currently megawatt wind turbines, the direct drive wind generators without gearbox, has high efficiency at low wind speed, low noise, high life expectancy, reduced crew volume, lower operation and maintenance costs, and many other advantages [1,2].

Direct drive variable speed constant frequency (DDVSCF) wind power system shows in Figure 1 [3], the wind wheel synchronous generator connected directly without raising speed gearbox. The wind energy transforms into AC which frequency, amplitude, phase are all change with time and then convert into DC by converter, and then converted by the inverter to a three-phase alternating current of constant amplitude and constant frequency to the grid. The active and reactive power of system is controlled by the power electronic converters to tracking the maximum power point and the maximum efficient use of wind energy [4-7].

The generator in Direct drive wind power generation system (DDWEGS) are two main types: rotor electric field concentrated winding rotor permanent magnet synchronous generator and excitation permanent magnet synchronous generators. Concentrated winding rotor electric field type synchronous generator (DDWEGS) needs to provide excitation current to rotor which needs slip rings and brushes. The failure rate of slip rings and 


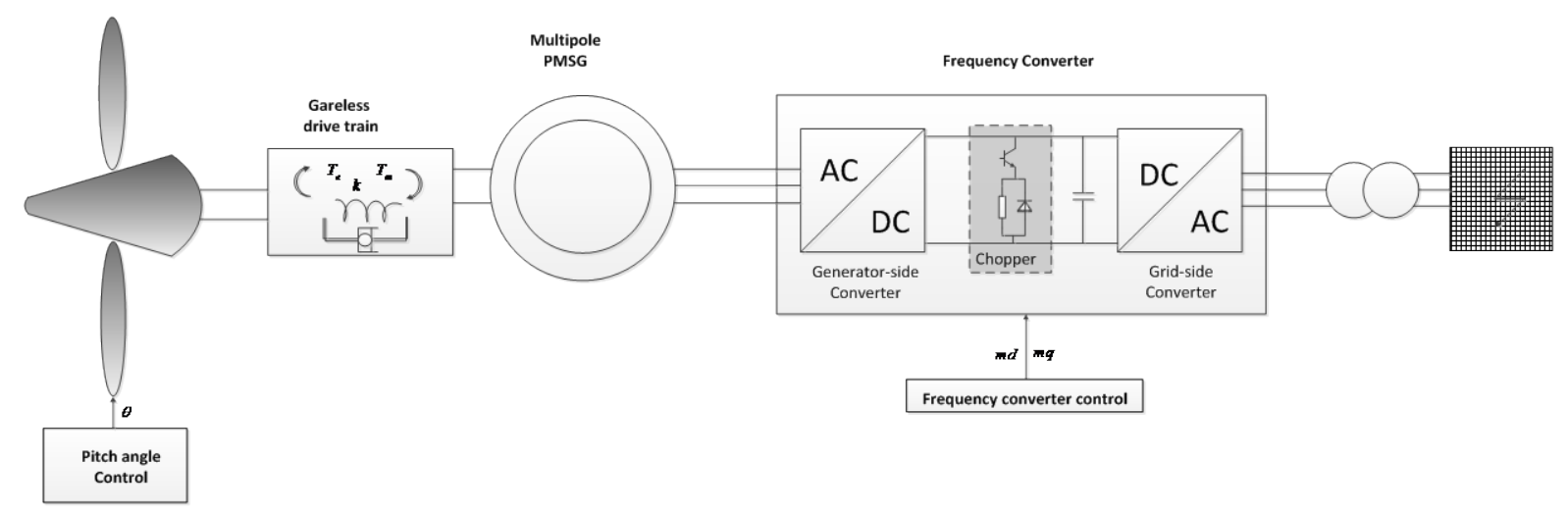

Figure 1. The diagram of PMSG wind energy generation system.

brushes are high, and require periodic replacement. The permanent magnet rotor with permanent magnet excitation for DDWEGS material to build the rotor field, eliminating the need for slip rings, brushes and gear boxes and other devices without regular maintenance, it has compact system architecture, high machine reliability and operating efficiency.

Direct drive wind power generation system using permanent magnet synchronous generator, without excitation control, It has wide range of motor operating speed and high motor power density, smaller size.

Some direct-drive that combined wind wheel with external rotor, eliminating the hub, rotor blades mounted directly outside, further simplifying the structure for reducing the weight .

\section{Chopper Circuit Hardware Connections}

When the system is functioning properly, the ChopperIGBT shuts down, and Chopper circuit does not access system. When a fall happens to the system voltage, DC input power exceeds output power. That will make the DC voltage increase, and threaten the safety of capacitor and inverter. At this time Chopper circuit conducts the IGBT, and put it into dump resistance. The excess energy is consumed by the dump resistance, so that the DC bus voltage can be stable [8]. Today there are two main structures of the Chopper circuit which can increase the dumping load in DC side. The dump resistances in Figure 2(a) connect directly to the DC side by IGBT while in Figure 2(b) dump resistances connect to the DC-side by Buck circuit. In Figure 2(a) the dump resistance connects directly to the high voltage DC bus, so the requirement of load voltage tolerance is high. When in Figure 2(b) dump resistance passes Buck step-down circuit, it can make the requirement of the withstanding ability in resistance for the voltage lower, but the circuit adds the inductance component. When dump resistance cut, generating extra energy consumed by the dumping the resistance, so we need to ensure reliable heat dissipation; this paper mainly focuses on comparing two scenarios in Figure 2 [3,9].

\section{Dumping Direct-Drive Permanent-Magnet Synchronous Motor Resistor Value}

In the process of circuit failure, since the power imbalance caused by the external network power voltage drop, DC bus capacitor voltage rises, at this time by putting Chopper circuit can consume excess active in order to maintain the DC voltage.

When converter losses are ignored, the difference between the active $P_{g e n}$ and $P_{g r i d}$ of the output of rectifier and inverter is [10]

$$
\frac{1}{2} C \frac{d U_{d k}^{2}}{d t}=P_{g e n}-P_{g r i d}=\Delta P
$$

After input dump resistance, DC voltage shall be maintained in the voltage within the range of values allowed, so dump resistance $R_{d}$ should read as follows:

$$
R_{d}=U_{d \mathbf{m a x}}^{2} / \Delta P
$$

$U_{d c m a x}$ is the maximum voltage allowed by DC bus. Considering the Depth of voltage dips, Network-side converter overload, the maximum allowable voltage DC and other factors, the Theoretical upper limit for dump 


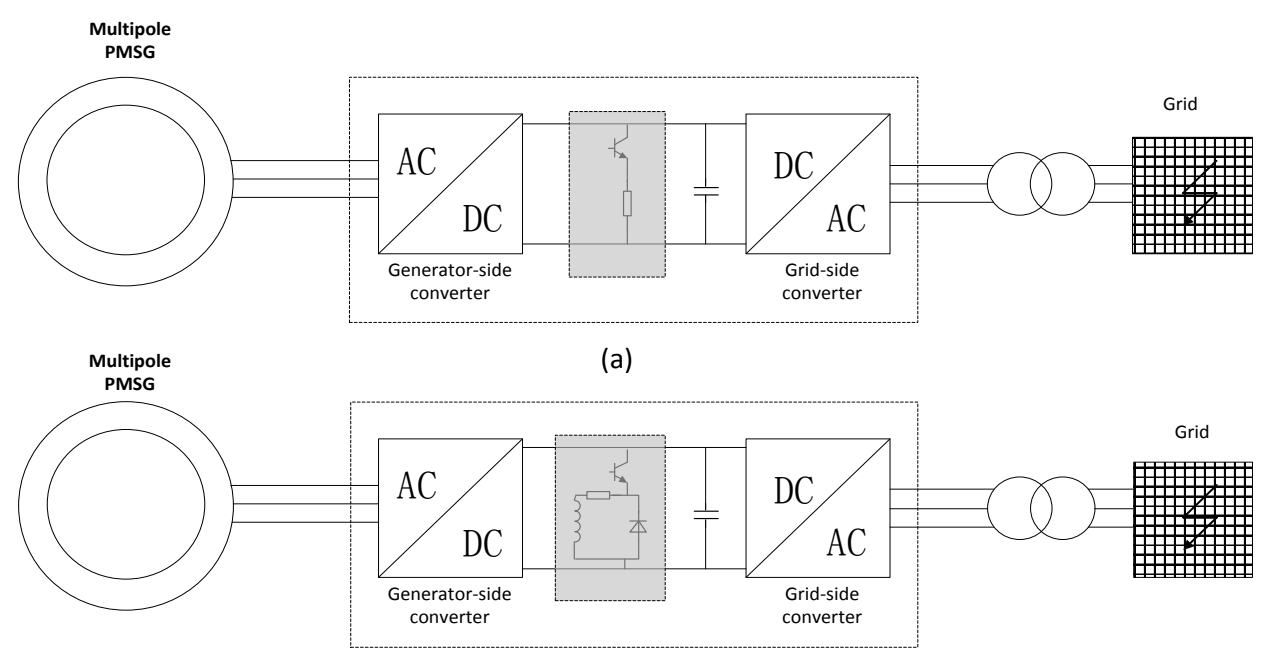

(b)

Figure 2. Chopper circuit scheme.

resistance is:

$$
R_{d \max }=\frac{U_{d c \max }^{2}}{P_{N}-3 \alpha u_{g}(1+\beta) i_{g}}
$$

In the formula: $P_{N}$ is unit rated activity; $\alpha$ is the ratio of fault voltage and rated voltage; $u_{g}$ is rated voltage famous value; $i_{g}$ is network-side converter rated current known values; $\beta$ is the proportions of exceeds its rating when goes through the network side converter current.

Dump resistance may burn when through the large current; therefore we must ensure that the resistor temperature rise is less than the maximum temperature rise. The former is equal to the cooling temperature resistance $\Delta T$ plus the relative cooling of the temperature rise $\Delta T_{2}$. By heat balance equation,

$$
\Delta T_{1}=p /(q c)
$$

In the formula: $P$ is the dump resistance power consumption, w; $c\left(W \cdot s /\left(m^{3 \circ} C\right)\right)$ is the specific heat; $q$ $\left(\mathrm{m}^{3}\right)$ is the cooling medium volume, The unit of $\Delta T_{1}$ is ${ }^{\circ} \mathrm{C}$

Dump resistance cooling is the heat exchange of forced Convection and thermal conduct at the same time, according to the formula for convective heat transfer,

$$
\Delta T_{2}=P_{1} /(\delta A)
$$

$\Delta T_{2}\left({ }^{\circ} \mathrm{C}\right)$ is the convective heat transfer between the two interfaces temperature difference, that is the resistance temperature of the surrounding cooling medium; $P_{1}$ is the overall heat transfer in terms of dump resistance power consumption. Make $P_{1}=\theta P, \theta$ is conversion Factor; $\delta\left(\mathrm{kW} /\left(\mathrm{m}^{3} \cdot{ }^{\circ} \mathrm{C}\right)\right)$ is heat transfer coefficient; $A\left(\mathrm{~m}^{2}\right)$ is heat transfer area, and it is the entire surface area of dump resistance, Therefore, the dump resistance temperature-rise is:

$$
\Delta T=\Delta T_{1}+\Delta T_{2}=p_{\max }\left(\frac{1}{q c}+\frac{\theta}{\delta A}\right) \leq \Delta T_{\max }
$$

According the formula (6), we can get the maximum allowed power of dump resistance is $P_{\max }$, according to $P_{\max }$, the dump resistance limit is:

$$
\frac{U_{d c}^{2}}{P_{\max }} \leq R_{d}
$$

According to resistance range from (8) to (12), when there is a serious failure in the grid, the direct drive unit is not off-grid. 


\section{The Equivalent Circuit Model of Chopper and Its Simulation}

We use a hybrid power system electromagnetic transient simulation program DigSILENT/Power Factory to build the model, and simulate the transient process PMSG wind turbines in the case of a three-phase short circuit. Make comparison of equivalent model of transient stability of wind turbines in different chopper circuit state [5,11-14].

The capacity of PMSG in the DigSILENT model in this paper is $1.5 \mathrm{WM}$, and the inverter capacity is 2.5 MVA, which is $166.6 \%$ of PMSG capacity. Rated voltage of DC side is $5.4 \mathrm{KV}$, rated voltage of PMSG stator side is $3.3 \mathrm{KV}$, network-side converter outlet voltage $3.31 \mathrm{KV}$, through the transformer and the power line to $220 \mathrm{KV}$. During normal operation, the output is active PMSG 1.5 WM, reactive power is 0.113 MVar.

\subsection{The Impact of Dump Resistance Value of Chopper to the Direct Drive Wind Power Generation System}

By formula (8) calculated in the model ranges in dump resistance to between $4.7 \Omega$ and $20.7 \Omega$. We take 5 , 10 , 15, 20 four experiments to simulation results are as follows in Figure 3.

It can be seen that the DC voltage has the most stable voltage when dumping the resistance value at around $15 \Omega$, thus it can achieve the best effect of the low voltage ride through (LVRT).

\subsection{The Impact of Chopper with Buck Circuit to the Direct Drive Wind Power Generation System}

This section experiments, set a three-phase short circuit grid fault at PCC as $1 \mathrm{~s}$, last $0.1 \mathrm{~s}$. When DC voltage reaches $5670 \mathrm{~V}(1.05 \mathrm{pu})$, the Chopper circuit takes action. The dump resistance is 15 ohm, Chopper circuit connection using Figure 2(b) in connection with the inductor. Simulation case is shown in Figure 4.

It can be seen that, due to the effect of inductive, the peak voltage of the DC bus during fault is reduced. However, it would have a bad impact on DC-bus when the inductance is too large.

\section{Summarize}

This paper studies the direct-drive permanent magnet synchronous machine in low voltage ride through. Especially Chopper circuit topologies and unloading resistance value, the main conclusions are as follows:

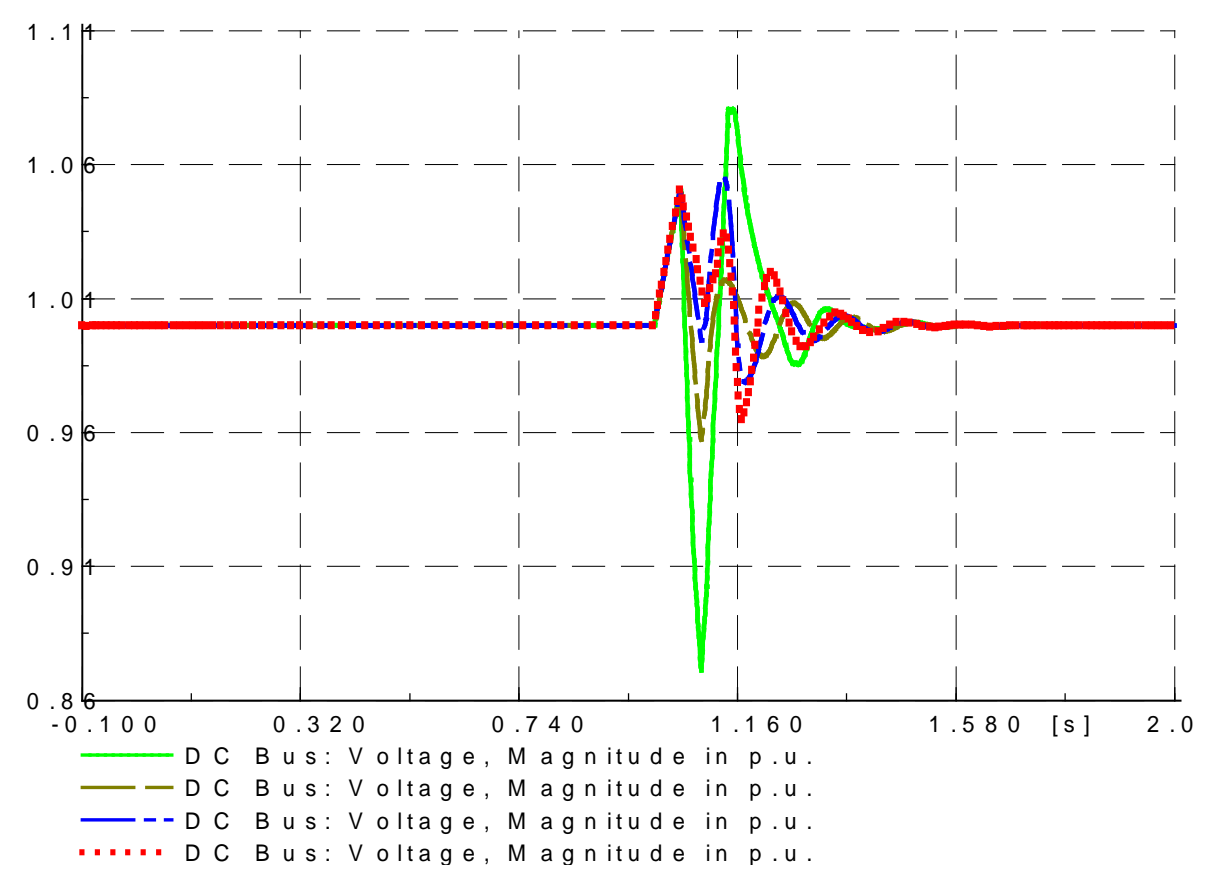

Figure 3. The DC-link wave of direct-drive wind energy generation system with different resistances in fault. 


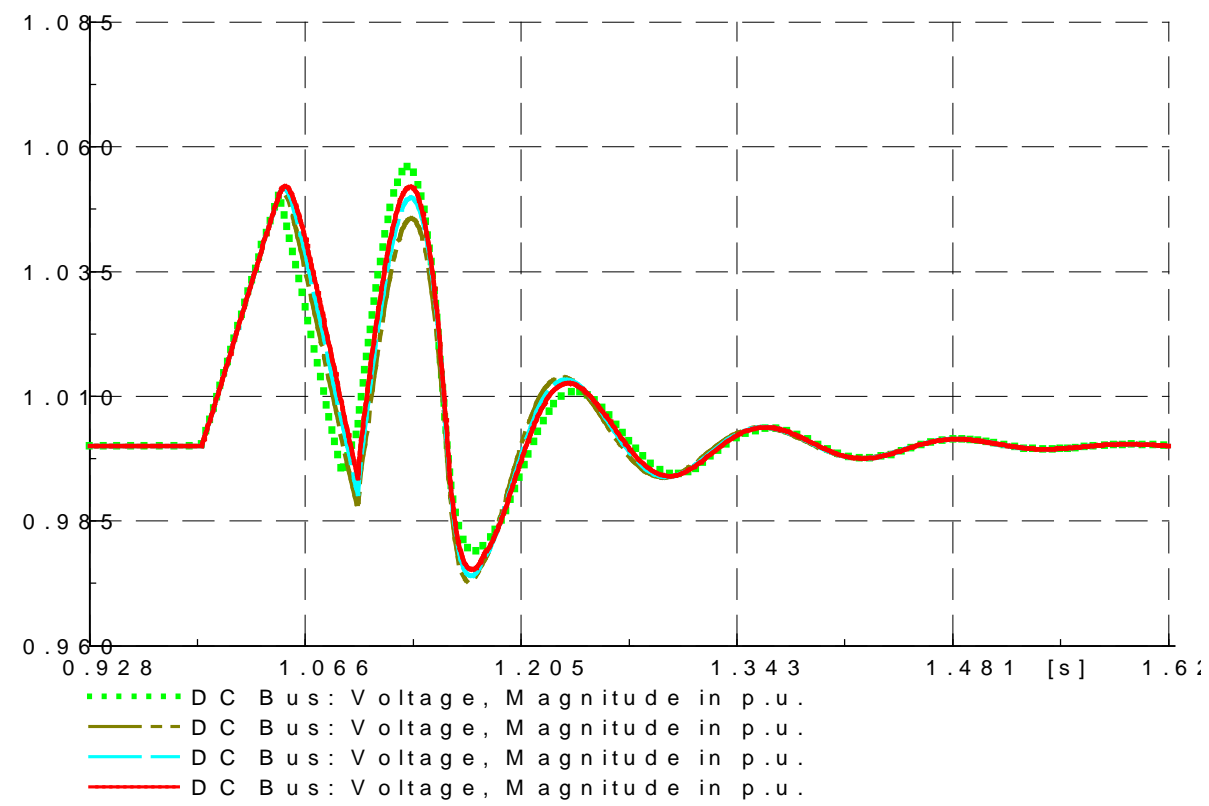

Figure 4. The DC-link wave of direct-drive wind energy generation system with different reactance in fault.

1) The difference of dump resistance, inductance and the way connected to Chopper circuit has different effects on low voltage ride through capability.

2) Due to the buffering effect of the inductor, Chopper circuit with Buck Chopper circuit has more excellent results of low voltage ride through than that with only resistance. Accordingly, the use of direct-drive wind power generation system with dumping Buck circuit or dual resistance is more significant to the improvement of low voltage ride through capability.

\section{References}

[1] Hansen, A.D. and Hansen, L.H. (2007) Wind Turbine Concept Market Penetration over 10 Years (1995-2004). Wind Energy, 81-97. http://dx.doi.org/10.1002/we.210

[2] Li, G.Q. (2007) Power System Transient Analysis. China Electric Power Press, Beijing.

[3] Zhang, M. (2008) Studies on the Modeling and Control System of Direct-Drive Permanent Magnet Synchronous Generator Based Wind Turbine. Master Thesis, Xi'an University of Technology, Xi'an.

[4] Zhang, X. (2008) Low Voltage Ride-Through Technologies in Wind Turbine Generation. Proceedings of the CSUEPSA, 20, 1-8.

[5] Akhmatov, V. (2003) Analysis of Dynamic Behavior of Electric Power Systems with Large Amount of Wind Power. Ph.D.Thesis, Rsted DTU, 1-260.

[6] Brando, G. Coccia, A. and Rizzo, R. (2004) Control Method of a Braking Chopper to Reduce Voltage Unbalance in a 3-Level Chopper. IEEE International Conference on Industrial Technology ICIT, 2, 975-978.

[7] Zhao, H.L., Yang, Y.X. and Wang, W.Q. (2010) Fault Ride-Through Simulation of Permanent Magnetic Synchronous Generator Connected into Power Grid. Power System and Clean Energy, 26, 15-18.

[8] Yang, Y.X., Liu, G.Q. and Du, B.X. (2012) Low Voltage Ride Through Technology Applied to Permanent Magnet Direct-driving Wind Generator Units. Journal of Electric Power, 27, 103-106.

[9] Xu, Q.H. and Cao, B.Z. (2012) Low Voltage Ride Through of 1.5 MW DFIG Converter under Symmetric Grid Failure. Dong Fang Turbine, 1-4.

[10] Li, S.H. (2013) Optimal Value and Group Switching Strategy of Dump Resistance for Direct-Drive Permanent Magnet Synchronous Generators. Power System Technology, 37, 1-7.

[11] Conroy, J. and Watson, R. (2009) Aggregate Modeling of Wind Farms Containing Full-Converter Wind Turbine Generators with Permanent Magnet Synchronous Machines: Transient Stability Studies. Renewable Power Generation, IET, 3, 39-52. http://dx.doi.org/10.1049/iet-rpg:20070091 
[12] Hansen, A.D. and Michalke, G. (2009) Multi-Pole Permanent Magnet Synchronous Generator Wind Turbines’ Grid Support Capability in Uninterrupted Operation during Grid Faults. Renewable Power Generation, IET, 3, 333-348. http://dx.doi.org/10.1049/iet-rpg.2008.0055

[13] Akhmatov, V. and Nielsen, H. (2003) Variable-Speed Wind Turbines with Multi-Pole Synchronous Permanent Magnet Generators. Part I: Modeling in Dynamic Simulation Tools. Windengineering, 27, 531-548. http://dx.doi.org/10.1260/030952403773617490

[14] Zhao, H.L., Wang, W.Q. and Wang, H.Y. (2010) Dynamic Characteristic Simulation of Permanent Magnetic Synchronous Generator Connected into Power Grid. Power System \& Automation, 32. 